Canadian Science Publishing
Michenom

Biochemistry and Cell Biology

Biochimie et biologie cellulaire

\title{
Functional assessment of MeCP2 in Rett syndrome and cancers of breast, colon and prostate
}

\begin{tabular}{|r|l|}
\hline Journal: & Biochemistry and Cell Biology \\
\hline Manuscript ID & bcb-2016-0154.R1 \\
\hline Manuscript Type: & Invited Review \\
\hline Complete List of Authors: & $\begin{array}{l}\text { PANDEY, SOMNATH; TEXAS TECH UNIVERSITY HEALTH SCIENCES } \\
\text { CENTER, IMMUNOLOGY \& MOLECULAR MICROBIOLOGY } \\
\text { PRUITT, KEVIN; TEXAS TECH UNIVERSITY HEALTH SCIENCES CENTER, } \\
\text { IMMUNOLOGY \& MOLECULAR MICROBIOLOGY }\end{array}$ \\
\hline Keyword: & MeCP2, Rett syndrome, colon cancer, breast cancer, prostate cancer \\
\hline \multicolumn{2}{|c}{} \\
\hline
\end{tabular}


1 Functional assessment of MeCP2 in Rett syndrome and cancers of breast, colon

2 and prostate

3 Somnath Pandey ${ }^{1}$ and Kevin Pruitt ${ }^{1 *}$

4

$5 \quad{ }^{1}$ Immunology and Molecular Microbiology, Texas Tech University Health Sciences

6 Center, Lubbock, TX, USA.

7

8

9

10

11 \#Address correspondence to: Kevin Pruitt, kevin.pruitt@ttuhsc.edu

12 Phone number: 806-743-2523

13 Fax number: 806-743-2334

14 *Present address: Immunology and Molecular Microbiology, Texas Tech University

15 Health Sciences Center, $36014^{\text {th }}$ Street, Lubbock, TX-79430, USA

16

17 Key Words: MeCP2, Rett, colon cancer, breast cancer, prostate cancer

18

19

20

21

22 


\section{Abstract}

24 Ever since the first report that mutations in methyl-CpG-binding protein 2 (MeCP2) causes Rett syndrome (RTT), a severe neurological disorder in females world-wide, there has been a keen interest to gain a comprehensive understanding of this protein. While the classical model associated with MeCP2 function suggests its role in gene suppression via recruitment of co-repressor complexes and histone deacetylases to methylated CpG-sites, recent discoveries have brought to light its role in transcription activation, modulation of RNA splicing and chromatin compaction. Various posttranslational modifications (PTMs) of MeCP2 further increase its functional versatility. Involvement of MeCP2 in pathologies other than RTT, such as tumorigenesis however, remains poorly explored and understood. This review provides a survey of the literature implicating MeCP2 in breast, colon and prostate cancer.

\section{Introduction}

MeCP2 is an X-chromosome associated gene, identified as the founding member of methyl-CpG binding domain (MBD) containing protein (Lewis et al. 1992). Our current knowledge regarding MeCP2 remains incomplete, despite its discovery some 24 years ago. The requirement of MeCP2 function is exemplified most notably in pathological conditions linked with RTT and other disorders namely classical autism, neonatal encephalopathy and X-linked mental retardation (Amir et al. 1999; Couvert et al. 2001; Lam et al. 2000; Beyer et al. 2002; Schanen et al. 1998). RTT involves an apparent normal development of female(s) upto 7-18 months of age during which they achieve the expected motor, social and language milestones, followed by regression of acquired skills (Hagberg et al. 1983; Hagberg and Witt-Engerstrom 1986). While RTT is most 
46 frequently seen in females than males (1 in every 15,000 females) (Hanefeld 1985), the

47 latter have a shorter lifespan and often develop fatal neonatal or congenital encephalopathy (Kankirawatana et al. 2006; Villard et al. 2000). MeCP2 is an epigenetic

49 reader that recognizes and binds to not only 5-methyl cytosine but also, 5hydroxymethyl-cytosine on DNA (Mellen et al. 2012). Recent works have implicated

51 MeCP2 in various cancers (Song et al. 2016; Muller et al. 2010; Ballestar et al. 2003).

52 Works from our own lab suggests the potential role MeCP2 plays in the progression of 53 colon cancer (Pandey et al. 2015).

54 Herein we begin with an introduction of MeCP2 followed by the multi-faceted functions it 55 is involved in, by interacting with various co-regulators or by undergoing multiple PTMs.

56 This review article examines the evidence for involvement of MeCP2 in the cancer of 57 the breast, colon and prostate. We stress the notion that MeCP2 is a key epigenetic 58 protein that is aberrantly regulated in cancer.

\section{MeCP2: Structure and function}

60 The pioneering works from Bird lab identified a nuclear protein, MeCP which 61 preferentially bound methylated DNA without any sequence specificity and was thought 62 to play a role in the inaccessibility of methylated CpG (Meehan et al. 1989; Meehan et 63 al. 1990). Later experiments from the same lab demonstrated that MeCP was actually 64 two distinguishable proteins - MeCP1 and MeCP2, with MeCP1 needing at least twelve 65 symmetrically methylated $\mathrm{CpG}$ shile MeCP2 was able to bind a single methylated $\mathrm{CpG}$ 66 base pair (Meehan, Lewis, and Bird 1992). Corroboration of the methyl-binding activity 67 of MeCP2 came from studies by Lewis et al. in 1992. They showed that MeCP2-LacZ 68 fusion protein was incapable of localizing to centromeric heterochromatin in methyl- 
transferase-deficient mouse cells. By constructing deletions of full length MeCP2, Nan et al. discovered the minimal region (MBD, $\sim 85$ amino acids long) required to bind DNA containing one or more symmetrically methylated CpGs. Additionally they also demonstrated that MBD binds to DNA as a monomer (Nan, Meehan, and Bird 1993).

Wakefield et al. in 1999, proposed the solution structure of recombinant MBD of MeCP2 via nuclear magnetic resonance (NMR) spectroscopy. They reported the structured core (amino acids 95-162) adopted a novel fold and formed a wedge shaped structure composed of a four-stranded anti-parallel $\beta$-sheet on one side and an $\alpha$-helix on the C-terminus end. Two anti-parallel $\beta$-strands are connected by an unstructured loop of five amino acid residues comprised of one positively charged and two polar residues (Wakefield et al. 1999). They also report that the DNA binding surface of MeCP2, the MBD, is rich in basic amino acids, lysine and arginine. The Bird lab recently published a high-resolution X-ray co-crystal structure of MBD bound to symmetrically methylated DNA (20 bp fragment of mouse brain-derived neurotrophic factor, BDNF) (Ho et al. 2008). Their model contradicted the previous proposed hypothesis that the stability of MBD-DNA interaction is brought about by hydrophobic association. The study revealed that the methyl-group predominately makes contact with tightly bound water molecules and that MeCP2's MBD, instead of recognizing the methylated CpG per se, interacts with the water molecules at the major groove of the methylated DNA (Ho et al. 2008).

Transcription repression domain (TRD) of MeCP2 was next characterized using an in vitro $\beta$-actin transcription assay, wherein the effects of fusion proteins made using Gal4 DNA binding domain and varying fragments of MeCP2 fragments were tested for 
92 their ability to repress a reporter gene construct containing Gal4 binding sites near the 93 promoter (Nan, Campoy, and Bird 1997). Rigorous experimentation led to the 94 identification of a minimal MeCP2 region encompassing amino acids 205-310, sufficient 95 to cause transcriptional silencing of a reporter gene construct. Both native and recombinant rat MeCP2 led to transcriptional repression in vitro from methylated 97 promoters but not from non-methylated promoters (Nan, Campoy, and Bird 1997). Recent findings from Huda lab have allowed for the characterization of two clusters of AT-Hook domains within MeCP2 (Baker et al. 2013). Such domains are found to be highly conserved among species and play a key role in DNA binding of chromatinassociated, non-histone proteins of the high-mobility group (HMG), where they were described for the first time (Reeves and Nissen 1990). Figure 1B shows the location of 103 AT-Hook domains in the two MeCP2 isoforms.

104 Recent investigations have identified intrinsic disorder in MeCP2 e2 isoform using 105 Foldlndex algorithm (Adams et al. 2007). Over a concentration range of $\sim 1000$ fold, $106 \mathrm{MeCP} 2$ is found to be monomeric in both low and high salt solutions. Circular dichroism 107 spectroscopy has predicted MeCP2 monomer to be $\sim 60 \%$ unstructured in conditions where it preferentially recognizes $\mathrm{CpG}$ dinucleotides and can form compact chromatin 109 (Adams et al. 2007). The same study carried out experiments involving protease digestion of MeCP2 and identified structurally distinct domains which are collectively 111 organized into tertiary structure possessing coil-like hydrodynamic properties, exhibiting 112 the intrinsic disorder in MeCP2 sequence. Both MBD and TRD when expressed as 113 individual fragments have the potential to function as non-specific DNA binding 
114 segments. The various structural attributes of MeCP2 provide a basis for its multi-

115 functional role in vitro and in vivo (Adams et al. 2007).

\section{MBD-dependent functions}

117 A schematic representation of the MeCP2 domains is shown in Figure 1B. In an effort to 118 discover protein factors mediating methyl-dependent repression, the same group used 119 co-immunoprecipitation (Co-IP) assays and showed that TRD interacts with a co120 repressor complex comprised of $\mathrm{mSIN} 3 \mathrm{~A}$ and histone deacetylases to result in the

121 alteration of chromatin structure via post-translational modifications of histone tails (Nan et al. 1998; Jones et al. 1998). These two studies were instrumental in demonstrating

123 that firstly, an indirect method exists by which MeCP2 can introduce alterations in 124 chromatin architecture by recruiting histone deacetylases, and secondly that DNA 125 methylation and histone deacetylation, the two global gene regulation mechanisms, can 126 be linked by MeCP2 (Figure 2), together with the finding that pharmacological inhibition 127 of deacetylase activity can partially relieve the methylation-dependent silencing. Such 128 partial alleviation of transcription repression was indicative of involvement of other 129 potential repressive modes.

130 MeCP2 interacts with various proteins to execute its functions in regulating cellular 131 physiology. In an in vitro myogenesis system, MeCP2 N-terminus has been shown to 132 associate with heterochromatin protein 1 (HP1) via Co-IP assays (Agarwal et al. 2007). 133 Using immunocytochemistry, the study reported that HP1 localization to 134 heterochromatin correlated with MeCP2 presence. HP1 is known to bind with H3K9me3 135 and interact with Suv39H1 methylatransferase (Yamamoto and Sonoda 2003), further 136 linking the presence of MeCP2 at heterochromatic regions. 
137 Maintenance DNA methyltransferase I (DNMT1) is known to interact with the TRD of 138 MeCP2 (Kimura and Shiota 2003), indicating that MeCP2 may potentially be involved in 139 faithful copying of methylation marks during DNA replication. Contrary to this, works 140 from (Nan, Meehan, and Bird 1993), underscored that binding to hemi-methylated DNA 141 of MeCP2 MBD, is negligible and so does not support the finding of hemi-methylated 142 DNA being bound by MeCP2.

143 Using Co-IP assays, Bird group found that methyl-binding domain of MeCP2 interacts 144 with $\alpha$-thalassemia/mental retardation, X-linked, ATRX protein (a SWI2/SNF2 DNA 145 helicase/ATPase that is mutated in ATRX syndrome) (Nan et al. 2007). They reported 146 that point mutations within the MBD of MeCP2 that caused RTT syndrome inhibited its 147 interaction with ATRX. In a DNA methylation-dependent manner, MeCP2, can recruit 148 the helicase domain of ATRX to heterochromatic foci in living mouse cells, allowing for a 149 tempting speculation that ATRX may be involved in the epigenetic regulation of genes 150 bound by MeCP2 (Nan et al. 2007).

A recent study revealed that MeCP2 is required for ATRX and CTCF binding to the H19 152 ICR and further suggested that MeCP2 and ATRX are involved in maintenance of nucleosome configuration conducive for CTCF binding to bring about regulation of gene 154 expression at a subset of imprinted domains (Kernohan et al. 2014). MeCP2 TRD binds c-Ski, encoded by the proto-oncogene c-Ski (Kokura et al. 2001). 156 Immunostaining analysis has demonstrated co-localization of MeCP2 and c-Ski in the 157 nuclear heterochromatic region. The magnitude of repression brought about by Gal4158 MeCP2 fusion protein was abolished following over expression of dominant negative 159 form of c-Ski. Also, antibody-mediated neutralization of Ski and Ski-related novel 
160 proteins, c-Ski and sno, led to the abrogation of transcription repression by Gal4161 MeCP2 which suggest a role of the ski gene family in methylation-dependent 162 transcription repression.

163 MeCP2 TRD and the amino-terminal domain were demonstrated to interact directly with 164 the ETS domain of PU.1 (ETS family of factors) by various pull-down and 165 immunoprecipitation assays (Suzuki et al. 2003). PU.1 transcriptional activity was found 166 to be repressed by MeCP2 on a reporter construct containing trimerized PU.1 binding 167 sites and that this transcription suppression can be rescued by TSA, a histone 168 deacetylase inhibitor. By performing pull-down assays, it was discovered that MeCP2 169 preferentially associates with the complex containing PU.1-mSIN3A-HDAC1 and not 170 with PU.1-CBP. ChIP-PCR assays using undifferentiated murine erythroleukemia cells 171 (MEL) demonstrated that MeCP2 and PU.1 are enriched at PU.1-binding site present on 172 the reporter construct and that present in the intron (intervening sequence 2 region) of $173 \beta$-globin gene, known to regulate its gene expression. It was also shown that during the 174 course of erythroid differentiation of MEL cells, PU.1-mSIN3A-HDAC1 complex 175 disappeared from this region (Suzuki et al. 2003). Thus by forming a complex with 176 mSIN3A and HDAC1, MeCP2 can repress PU.1. While underscoring the multi177 functional nature of this protein, a recent review describes the various protein factors 178 MeCP2 associates with (Guy et al. 2011). Figure 3 illustrates the minimal region 179 required for the interaction of different candidate binding partners with MeCP2 e2 180 isoform.

\section{MeCP2 Isoforms}


182 Our understanding of MeCP2 location, expression patterns, and functionality increased 183 with the discovery of a previously unknown isoform MeCP2B (Mnatzakanian et al. 184 2004). The new isoform e1, or MeCP2B, is a splice variant whose translation start site 185 begins at exon 1 of MeCP2, foregoes exon 2 and encompasses full-length exons 3 and 1864 to give rise to a 498 amino acid long protein, while the known isoform e2 or MeCP2A 187 begins at exon 2, followed by full-length exons 3 and 4 to yield a 486 amino acid protein 188 (Figure 1). Hence the two isoforms differ at their N-terminus. MeCP2 e2 is expressed in 189 skeletal muscle, liver, spleen and prostate while MeCP2 e1 is expressed primarily in 190 fetal and adult brain and brain sub-regions. In the adult human brain, MeCP2 e1 191 expression is ten times the expression of MeCP2 e2 (Mnatzakanian et al. 2004). These 192 findings indicate that important differences between these two isoforms remain to be 193 identified.

194 MeCP2 post-translational modifications (PTMs)

195 MeCP2 PTMs make possible the intricacies of MeCP2 transcriptional modulation. While 196 many PTMs of MeCP2 have been found to date, phosphorylation of MeCP2 remains the 197 most well studied. MeCP2 phosphorylation was first noted in 2003 when Chen et al 198 (Chen et al. 2003) found that MeCP2 selectively binds to the promoter III of BDNF gene 199 and functions to negatively regulate its expression. They discovered that membrane 200 depolarization of rat cortical neurons in response to increased $\mathrm{KCl}$ triggers calcium201 dependent phosphorylation and exiting of MeCP2 from BDNF promoter and facilitates 202 chromatin remodeling to allow for BDNF transcription. Hence, MeCP2 is pertinent in 203 regulating genes involved in neuronal activity and the dysregulation of that role might be 204 the causative factor for RTT. 
205 De novo phosphorylation of MeCP2 at S421 (serine residue at 421 position) in cultured 206 rat neurons, triggered by neuronal activity followed by an influx of calcium was reported 207 in 2006 (Zhou et al. 2006). In this study, it was demonstrated that phosphorylation of 208 MeCP2 at serine 421 residue was selectively induced in vivo in response to synaptic 209 activity driven via either light exposure or via pharmacologically induced seizures in 210 rodents. Phosphorylation at this site results in de-repression of BDNF transcription and 211 regulates MeCP2-dependent dendritic growth and spine maturation. Western blot 212 analysis from whole cell extracts prepared from a 4-week old male mouse revealed that 213 ser421 phosphorylation occurred specifically in the brain and not in any other tissue 214 analyzed. These observations indicate that by inducing phosphorylation of MeCP2, 215 neuronal activity orchestrates a gene expression program to bring about nervous 216 system maturation and the disruption of which may underlie the RTT phenotype in 217 individuals with MeCP2 mutation.

218 Mass spectrometry analysis has shown serine 80 residue of MeCP2 to be 219 phosphorylated in rodents either basally or when subjected to seizures (Tao et al. 220 2009). Neuronal activity triggers dephosphorylation of ser80 while S80A mutant 221 contributes to reduced association of MeCP2 to several gene promoters and 222 concomitantly altering their expression (Tao et al. 2009).

223 Using a dephosphorylation mimetic MeCP2 S421A mouse, it was shown in vivo, that 224 loss of ser421 phosphorylation leads to an abnormal development of dendrites and 225 synapse, and a defective behavioral response to new experience (Cohen et al. 2011), 226 suggesting that RTT is a disorder of experience-dependent neuronal development. 
227 Studies using tandem mass spectrometry (MS/MS) have reported the presence of 228 multiple MeCP2 PTMs, viz., phosphorylation, acetylation and ubiquitination (Gonzales 229 et al. 2012) in SH-SY5Y cells ectopically expressing MeCP2. Gonzales et al. discovered 230 that phosphorylation at S80 or S229 residue of MeCP2 selectively alters interactions 231 with chromatin factors such as SMC3 and HP1, and co-factors such as SIN3A and YB232 1, in vivo. MeCP2 phosphorylation was found to be indispensable to bring about 233 differentiation-induced activation and/ or repression of receptor tyrosine kinase (RET), 234 and early growth response factor 2 (EGR2), MeCP2 target genes (Gonzales et al. 235 2012). It is worth mentioning that MeCP2 was found to be acetylated at lysine residues 236305,307 and 321.

237 Phosphotryptic mapping was utilized in 2013 to identify three novel MeCP2 238 phosphorylation sites (S86, S274, and T308) following neuronal stimulation (Ebert et al. 239 2013). Various agents such as those that increase intracellular cAMP level, neuronal 240 activity, or BDNF, can differentially induce phosphorylation of these sites, suggesting 241 the epigenetic regulatory role of MeCP2 of gene expression, by integrating various 242 environmental signals. T308 phosphorylation was shown to prevent the interaction of 243 MeCP2 TRD and the nuclear receptor-corepressor (NCoR) complex and hence 244 decreasing MeCP2's ability to cause transcription repression (Ebert et al. 2013). 245 Neuronal stimulation in knock-in mice harboring a typical human RTT missense 246 mutation R306C, failed to elicit T308 phosphorylation, indicating that loss of MeCP2 247 T308 phosphorylation may potentially contribute to RTT. Ebert et al., further showed 248 that mice bearing MeCP2 T308A mutation exhibited reduced induction of a subset of 249 neuronal activity-regulated genes and to RTT-like phenotype. Hence, neuronal 
250 stimulation-dependent T308 phosphorylation regulates MeCP2's interaction with the

251 NCoR complex and that RTT phenotype in humans could be attributed, in part, to the 252 failure of activity-dependent phosphorylation of MeCP2 at T308 residue and the 253 subsequent loss of phosphorylation-controlled MeCP2-NCoR complex interaction. 254 Figure 3 illustrates the distribution of different phosphorylation sites over MeCP2 255 domain map. Excellent reviews have highlighted the role of multiple other MeCP2 256 phosphorylation sites (Bellini et al. 2014; Ausio, Martinez de Paz, and Esteller 2014).

257 The role of protein acetylation in regulating cell physiology is becoming increasingly 258 appreciated (Yang and Seto 2008). Recent studies have reported MeCP2 to be 259 acetylated (Choudhary et al. 2009; Zocchi and Sassone-Corsi 2012; Gonzales et al. 260 2012; Pandey et al. 2015), and because little is known about how acetylation influences 261 MeCP2 function, it remains an active area of research. Over expression of SIRT1 262 deacetylase was shown to result in reduced acetylation of mouse MeCP2 e1 isoform at 263 lysine 464 (K464) or human lysine 461, when MeCP2 was ectopically expressed in 264 HEK293 cells (Zocchi and Sassone-Corsi 2012). ChIP-qPCR analysis of hippocampus 265 tissue revealed that MeCP2 enrichment at exon IV of BDNF was significantly increased 266 in SIRT1 ${ }^{\Delta \mathrm{ex} 4}$ mice relative to wild-type control mice and was linked to decreased BDNF 267 expression in SIRT1 ${ }^{\Delta \mathrm{ex} 4}$ mice (Zocchi and Sassone-Corsi 2012).

268 In an attempt to identify endogenously acetylated lysine(s) in MeCP2 in multiple cancer 269 cell line models, we pharmacologically inhibited SIRT1 and using tandem MS/MS, 270 identified various acetylated lysines (K22, K135, K171, K200, K256, K271 and K289)

271 (Pandey et al. 2015). Our acetylation mimetic mutant, MeCP2 K171Q, elicited 272 decreased binding to chromatin remodeling proteins, ATRX and HDAC1, as compared 
273 MeCP2-WT and was associated with retarded growth and proliferation of RKO colon 274 cancer cells in which it was over expressed (Pandey et al. 2015). Figure 3 illustrates the 275 distribution of different acetylation sites over MeCP2 e2 isoform domain map.

276 Sumoylation of MeCP2 has been found to occur on K223, that brings about the 277 recruitment of deacetylase complex HDAC1/2 and the deacetylation mimetic mutant, $278 \mathrm{~K} 223 \mathrm{R}$, inhibits transcriptional repression function of MeCP2 in primary cortical neurons 279 (Cheng et al. 2014). Furthermore, sumoylation at K223 plays a pertinent role in 280 regulating the formation of excitatory synapse.

281 Many other MeCP2 PTMs such as the methylation and ubiquitination of lysine residues 282 have been reported elsewhere (Bellini et al. 2014). The above mentioned PTMs of 283 MeCP2 elevate its ability to function dynamically within various cells of the body, 284 thereby necessitating the need to characterize other modifications of MeCP2.

285 TRANSCRIPTIONAL ACTIVATOR

286 While attempting to delineate the molecular mechanism(s) fundamental to 287 neuropsychiatric disorders resulting from either MeCP2 depletion or over-expression 288 (clone including regions responsible for both $\mathrm{e} 1$ and $\mathrm{e} 2$ isoforms) in mice while 289 analyzing patterns of gene expression in mouse hypothalamus, it was observed that, 290 although in both cases, the expression levels of thousands of genes was altered, 291 majority of the genes ( $85 \%$ ) were surprisingly upregulated by MeCP2 (Chahrour et al. 292 2008). MeCP2 was found to interact with the transcriptional activator, cyclic-AMP 293 response element binding protein 1 (CREB1) while occupying the promoter of an 294 activated target gene did not interact with the same while occupying a repressed target 295 gene promoter. Interestingly, findings from the same lab later extended the observation 
296 in the cerebellum, wherein a multitude of genes were found to be upregulated in 297 MeCP2-Tg mice (over-expressing MeCP2) and downregulated in MeCP2-null mice 298 consistence with MeCP2's role as a transcriptional modulator capable of both increasing 299 and decreasing the expression of genes (Ben-Shachar et al. 2009). Such investigations 300 are indicative that gene expression alterations in various regions of the brain occur 301 following MeCP2 loss or gain of function. The role of MeCP2 as a modulator has been 302 recently review by others (Delcuve, Rastegar, and Davie 2009). Figure 4 illustrates how 303 MeCP2 can function as a transcriptional activator.

304 REGULATION OF RNA SPLICING

305 Examples mentioned above provide insights to the various roles MeCP2 plays by 306 interacting with multiple proteins. MeCP2 e2 isoform can associate with DNA/RNA 307 binding protein, Y-box binding protein (YB-1) and regulate reporter minigene splicing 308 (Young et al. 2005). The same group further demonstrated that RNA mediates 309 interaction between YB-1 and MeCP2 and that this interaction was not disrupted when 310 R106W-MeCP2 (RTT mutant form of MeCP2) was used or a functional methyl-binding 311 domain of MeCP2 is not absolute for its interaction with YB-1. Such results together with 312 the findings that a mouse model of RTT (MeCP2-308 mutant) shows abnormal 313 alternative splicing pattern as compared to MeCP2-WT (Young et al. 2005), is indicative 314 of MeCP2's role in regulating splicing.

315 It was later reported that MeCP2 is increasingly present at the heavily methylated 316 alternatively spliced exons (ASEs) and that inhibition of DNA methylation results in 317 firstly, abnormal splicing of ASEs and secondly, disrupts the methylation specific 318 targeting of MeCP2 (Maunakea et al. 2013). Genetic ablation of endogenous MeCP2 
319 was found to cause increased acetylation of histone H4 and abnormal skipping events

320 associated with ASEs which over-lapped to a great degree with the skipping of exons

321 observed when HDACs were pharmacologically inhibited. These findings thus suggest

322 that intragenic CpG-methylation enhances recognition of exons by serving as a docking

323 site for MeCP2 which in turn brings about the recruitment of HDACs to maintain the

324 local hypoacetyation of histones to modulate alternative RNA splicing.

325

\section{MBD-INDEPENDENT FUNCTIONS}

326 A rapid increase in MeCP2 research has occurred ever since the discovery that MeCP2

327 is an etiological factor for Rett syndrome (RTT) (Amir et al. 1999). As opposed to the

328 earlier methyl-binding activity of MeCP2, recent findings uncover its ability to bind to

329 chromatin and DNA independent of CpG-methylation status (Georgel et al. 2003;

330 Nikitina et al. 2007; Yasui et al. 2007; Chahrour et al. 2008; Ben-Shachar et al. 2009).

331 Studies discussed below involve the usage of MeCP2 e2 isoform.

332 In vitro studies demonstrating histone deacetylase-independent compaction via

333 formation of secondary and tertiary chromatin structures assembled by MeCP2 led to its

334 understanding as a complex nuclear protein, with a key role in globally regulating

335 chromatin architecture (Georgel et al. 2003). This study employed sophisticated

336 electron cryo-microscopy to characterize complexes formed between non-methylated

337 12-mer nucleosome arrays and recombinant human MeCP2, or specific missense

$338(\mathrm{R} 133 \mathrm{C})$ or non-sense (R168X) MeCP2 mutants and revealed the novel finding that 339 chromatin condensing region(s) of MeCP2 reside in domains other than the methyl340 binding domain. 
341 Such findings served as a foundation for a later study that utilized various truncated

342 forms of human MeCP2 to demonstrate that the methylation independent interaction

343 with nucleosome positioning-sequence was mediated by the C-terminal domain, and 344 also indicated a partial methyl-specific interaction being mediated via the MBD (Nikitina 345 et al. 2007). Results from electron microscopy analysis revealed the capability of 346 MeCP2 to modulate nucleosome array structure by trans-interaction with multiple arrays 347 and in-cis compaction of an array.

348 In an attempt to identify novel genes targeted by MeCP2, Horike et al., sequenced 349 MeCP2-binding sites in mouse brain and mapped an imprinted gene cluster on 350 chromosome 6 that contained two genes, Dlx5 and Dlx6 (Horike et al. 2005). These 351 genes showed two fold higher expression in MeCP2-null mice than those of MeCP2-WT 352 mice. Analyzing ChIP-coupled loop assays showed that MeCP2 mediated the formation 353 of silent chromatin-derived 11-kb chromatin loop at the Dlx5-Dlx6 locus and that this 354 loop was absent in MeCP2-null mice. While this indicates a new mechanism of MeCP2 355 driven gene regulation, recent works from a study has refuted against these findings 356 (Schule et al. 2007).

\section{Involvement in Breast Cancer}

358 One of the most common malignancies observed in women is breast cancer and it 359 remains one of the leading causes of mortality worldwide, despite the progress made in 360 the past 15-20 years (Yang et al. 2015; Murphy and Morris 2012).

361 An early report linking MeCP2 with breast cancer via in vitro studies showed that 362 partially methylating the promoter region of breast cancer predisposition gene BRCA1, 363 results in a MeCP2-dependent inhibition of gene expression (Magdinier et al. 2000). It 
364 was reported that dysregulation of MBD domain containing proteins MeCP2 and MBD2,

365 occurs in human breast cancer, following the quantification of their transcripts in normal

366 breast and in benign and neoplastic breast tumors via RT-PCR analysis (Billard et al.

367 2002).

368 A study in 2003 for the first time established a link between MeCP2 mRNA and 369 estrogen receptor status. The authors not only showed high MeCP2 mRNA expression 370 levels in neoplastic tissues than in non-neoplastic tissues, but also discovered higher

371 MeCP2 transcript levels in estrogen receptor positive $\left(\mathrm{ER}^{+}\right)$breast cancer samples than 372 those present in estrogen negative breast cancer specimen (Muller et al. 2003).

373 In an effort to demonstrate existence of gene specific binding or association pattern of 374 MBD proteins in cancer cells, Ballestar et al, showed that MeCP2 was one of the select 375 MBD proteins that was uniquely in association with Ras association domain family $1 \mathrm{~A}$ 376 gene (RASSF1A), ectonucleotide pyrophosphatase/ phosphodiesterase 4 (ENPP4), 377 phosphatidylinositol 4-phosphate 5-kinase (PIP5K) and retinoic acid receptor B2 gene 378 (RARB2) promoters (Ballestar et al. 2003). Such studies reveal that while certain 379 methylated CpG sequences interact with only one MBD protein, others can associate 380 either multiple or all of them. Thus, breast cancer involves MeCP2-dependent inhibition 381 of BRCA1 gene expression, together with elevated MeCP2 gene expression in 382 neoplastic tissues and increased localization of MeCP2 at the promoters of various 383 tumor suppressor genes (TSGs).

384 An investigation aimed at characterizing MeCP2 target sequences in MCF-7 breast 385 cancer cells, employed a preparative-ChIP assay to create a library containing MeCP2 386 bound sequences, such as the retrotransposons (mostly with Alu repeats), matrix- 
387 associated regions (MARs), CpG islands, and others (Koch and Stratling 2004). The study used multiple techniques including indirect immunofluorescence microscopy and concludes that the preference of MeCP2 at these regions is due to its sequence recognizing information potential (guanine nucleotide bases adjacent to $\mathrm{CpG}$ or $\mathrm{TpG}$ ) as demonstrated in earlier foot-printing assays (Weitzel, Buhrmester, and Stratling 1997). Epigenetic silencing of estrogen receptor- $\alpha$ (ER- $\alpha$ ) gene as a result of CpG hypermethylation correlates with the interaction and binding of MBD proteins including MeCP2, histone deacetylase 1 (HDAC1), and DNA methyl-transferases (DNMTs) proteins to the ER promoter in MDA-MB-231 breast cancer cells, and whose treatment with 5-aza-2'-deoxycytidine (inhibitor of DNMTs) and trichostatin A (inhibitor of HDAC activity) results in the vacating of the promoter by repressor complex comprising of MeCP2 and other MBD proteins, together with DNMTs and HDAC1 (Sharma et al. 2005). This treatment further correlates to increased transcription activation marks (H3Ac, H4Ac and H3K4me2) and decreased repressive marks (H3K9me2), leading to the re-expression of $E R-\alpha$ gene. Such studies involving the use of epigenetic drugs allows for a deeper understanding of the fundamental molecular mechanisms of chromatin remodeling resulting in ER reactivation. Findings from such studies aid in developing therapeutic strategies against breast cancer via epi-drugs. It was later shown that the repressive complex comprising of MeCP2, HDAC1 and DNMT1, while present at ER- $\alpha$ promoter in ER-negative MDA-MB-468 cells, remained absent at the same region in ER-positive MCF-7 cells (Rasti et al. 2012). MDA-MB-468 cells express a mutant p53 whose binding to HDAC1, DNMT1 and MeCP2 is not altered at ER- $\alpha$ promoter. Investigating $E R-\alpha$ gene silencing in these cells showed that down regulating 
410 DNMT1 or HDAC1 led to the disassembly of mutant p53 and the repressive complex

411 from ER- $\alpha$ gene promoter and this was consistent with the ER re-expression following

412 partial demethylation of ER- $\alpha$ promoter (Arabsolghar, Azimi, and Rasti 2013). HDAC1

413 plays a key role in ER- $\alpha$ gene silencing due to its deacetylase activity and for the

414 assembly of DNMT1 in the repressive complex. Also, mutant p53 binds to ER- $\alpha$ gene

415 promoter via direct association with HDAC1 and indirect association with DNMT1 and

416 MeCP2 in 468 cells (Arabsolghar, Azimi, and Rasti 2013).

417 An inhibitor of zinc-dependent deacetylases, valproic acid (VPA), brings about the 418 shutoff of ER- $\alpha$ promoter in a reversible manner via invoking recruitment of MeCP2 419 transcription repressor resulting in decreased levels of mRNA encoding ER- $\alpha$ and 420 subsequent loss of the protein in ER-positive breast cancer cells (Reid et al. 2005).

421 While characterizing epigenetic deregulation status in three breast cancer cells 422 representing different stages of human breast cancer, Tryndyak et al., showed 423 significant epigenetic alterations in breast cancer cells as compared to normal 424 mammary epithelial cells MCF-10-2A and demonstrated that the more malignant MDA425 MB-231 cells have a greater loss of CpG methylation followed by aberrant expression of 426 DNMT1, MBD proteins: MeCP2 and MBD2, reduced Suv4-20h2 leading to loss of 427 H4K20me3, and H4-hyperacetylation as compared to MCF-7 cells (Tryndyak, 428 Kovalchuk, and Pogribny 2006). Such results suggest that malignant breast cancer cells 429 are associated with greater epigenetic deregulation, including aberrant expression of 430 MeCP2, which may potentially be an indicative of aggressive tumor phenotype during 431 tumorigenesis. 
432 Epigenetic changes in mammary gland following exposure to ionizing radiation (IR) and 433 the induced carcinogenesis in a rat model of breast cancer has been studied. IR 434 exposure results in global DNA hypomethylation together with reduced levels of de novo 435 DNA methyltranseferase (DNMT3a and 3b), maintenance methyltranseferase (DNMT1) 436 and MeCP2 and linked to cell cycle control mechanisms and was in part associated to 437 activation of DNA repair pathway (Loree et al. 2006). Following irradiation, apoptosis 438 was significantly increased and was paralleled by a noticeable increase in cellular 439 proliferation.

440 Mechanistic studies involving malignant breast cancer have shown that kallikrein-related 441 peptidase 6 (KLK6), TSG silencing is due to formation of repressive chromatin mediated 442 by histone deacetylation and $\mathrm{CpG}$ methylation-dependent binding of MeCP2 at its 443 proximal promoter (Pampalakis et al. 2009). Stable overexpression of KLK6 in MDA444 MB-231 cells exhibits reversal of malignant phenotype i.e., decreased proliferation 445 rates, reduced cellular motility, inhibition of anchorage-independent growth, and 446 reduced in vivo orthotopic tumor formation in severe combined immunodeficiency 447 (SCID) mice. This suggests that via potential inhibition of epithelial-to-mesenchymal 448 transition (EMT), KLK6 might exert a protective role against metastatic breast cancer.

449 Utilizing a luciferase assay, it has been shown that in MCF-7 cells, MeCP2 can repress 450 select target gene promoters namely, CD44, phosphatase and tensin homologue 451 (PTEN), Cyclin D2 and glioma-pathogenesis related protein 1 (GLIPR1) (Muller et al. 452 2010).

453 Bisulfite sequencing analysis revealed that leukemia inhibitory factor (LIF) regulating 454 progression of various types of cancer, is hypermethylated within the promoter region, 
455 in normal breast epithelial cells but highly demethylated with cancer progression (Shin, 456 Park, and Jang 2011). In line with this observation, enrichment of MeCP2 and 457 H3K9me2 decreased with breast cancer progression while H3K4me2 activation mark 458 increased inversely together with increased binding of Sp1 transcription factor at such 459 hypomethylated sites. Genetic ablation of LIF lead to substantial reduction of cellular 460 growth and colony formation in breast cancer cells analyzed (Shin, Park, and Jang 461 2011).

462 Immunohistochemistry analysis reveals MeCP2 protein to be significantly higher in 463 invasive ductal carcinoma (IDC) specimen than in non-neoplastic lesions (Xu et al. 464 2012).

465 Upregulated in breast cancer, a disintegrin and metalloprotease domain-containing 466 protein $12(A D A M-12)$ is subjected to transcriptional suppression by Z-DNA-forming 467 negative regulatory element (NRE) present at its 5'-UTR, facilitates binding to 468 epigenetic regulator, MeCP2 and nuclear family of transcription factors, NF1C and 469 NF1X, contributing to its low expression in normal cells and tissues (Ray et al. 2013).

470 Natural compounds including curcumin, epigallocatechin gallate (EGCG), resveratrol, 471 and others have been shown to cause reversal of epigenetic modification by reducing 472 the protein expression of DNMT1, MeCP2 and HDAC1 (Mirza et al. 2013).

473 Anti-cancer peptide SA12 binds to MeCP2 in vitro and results in upregulation of p53 and 474 PTEN expression and further inducing apoptosis by targeting $\mathrm{Bcl}-2$ family and related 475 caspases in SKBr-3 cells (Yang et al. 2015).

476 Analysis of $~ 9221$ human tumor samples from TCGA has allowed for the finding that 477 MeCP2 is a frequently amplified oncogene in a number of tumors including triple 
478 negative breast cancer (TNBC) (Neupane et al. 2016). Over-expressing MeCP2 e2 479 isoform in human mammary epithelial cells (HMECs) enables anchorage independent 480 growth to a level almost similar to that resulting from an infection with activated HRAS 481 while the longer e1 isoform fails to enable such growth (Neupane et al. 2016). The 482 same study further showed that while both the isoforms could activate PI3K pathway, 483 only MeCP2 e2 isoform is capable of activating MAPK pathway in HMECs during 484 growth factor deprivation. Such investigations have revealed MeCP2 functionality in 485 different cellular contexts.

486 Above mentioned studies underscore the alteration in epigenetic process in ER-positive 487 and TNBC cells and the role MeCP2 plays in silencing TSGs or promoting cancer 488 survival.

489 Involvement in Colon cancer

490 Strong E-cadherin (ECAD) TSG expression and a lack of promoter CpG methylation in 491 normal colonic mucosa and other well-differentiated adenocarcinoma has been 492 observed, while both mucinous adenocarcinoma and signet-ring cell carcinoma display 493 complete $\mathrm{CpG}$ methylation and strong MeCP2 expression patterns when 29 cases of 494 colorectal carcinoma were analyzed in Indonesia (Darwanto et al. 2003). Loss of 495 MeCP2 expression was found to be correlated to ECAD re-expression.

496 Patients with RTT are known to have gastrointestinal pathologies, owing to loss of 497 parasympathetic control. A recent study reported the risk of colon cancer in a RTT 498 patient (Yilmaz et al. 2014). Genome-wide studies in YB5 cells (a clonal derivative of 499 the SW48 colon cancer cell line), demonstrated that re-expression of basally silenced 500 and hypermethylated genes by the combinatorial action of carboplatin and decitabine 
501 was significantly better than when induced by the two drugs alone. The combination 502 also brought about the inhibition of HP1a expression accompanied by increase in $503 \mathrm{H} 3 \mathrm{~K} 4 \mathrm{me} 3$ and $\mathrm{H} 3 \mathrm{~K} 9 \mathrm{ac}$ at the reactivated gene promoters together with decreased 504 binding of MeCP2 and MBD2 (Qin et al. 2015). Hence the epigenetic synergy shown by 505 the combination of a platinum analog, carboplatin, and decitabine can be used treat 506 various cancers.

507 As mentioned earlier, specifically targeting SIRT1 deacetylase by pharmacological 508 drugs, we discovered MeCP2 to be endogenously acetylated in RKO colon cancer cells 509 (Pandey et al. 2015). K171 residue identified via mass spectrometry analysis as an 510 acetylated site was particularly interesting as it was conserved from Xenopus species to 511 Homosapiens. Chromosomal associated proteins such as HDAC1, MeCP2 and ATRX 512 are known to bring about chromatin remodeling including repression of TSG expression. 513 We observed reduced binding of ATRX and HDAC1 to our acetylation mimetic mutant, 514 MeCP2 K171Q (Pandey et al. 2015). A different approach involving the pharmacological 515 inhibition of SIRT1 too showed consistent result. Transient and stable over expression 516 of K171Q mutant in colon cancer cells showed retarded growth and proliferation as 517 compared to those over expressing MeCP2-WT. This shows the role of specific MeCP2 518 lysine residue in regulating cell physiology and growth characteristics.

519 A recent study demonstrated that genetic ablation of MeCP2 in DLD-1 colorectal cancer 520 cells results in cell cycle arrest at G0/G1 phase, decrease in cell viability, and also leads 521 to inhibition of cell migration (Song et al. 2016). Results from this study that inhibition of 522 MeCP2 function in DLD1 cells leading to decreased cell growth are in consensus with 523 our study of MeCP2 K171Q mutant showing retarded growth in RKO cells. This 
524 suggests that MeCP2 may serve as a potential target towards treating colorectal 525 cancer.

526 In an unbiased screen for identification of drugs which reactivated basally suppressed

527 genes in colon cancer cells, led to the identification of classical epigenetic drugs 528 (inhibitors of DNA methylation and histone deacetylation) and 11 other pharmacological 529 drugs which led to induction of methylated-CpG island promoters of not only 530 endogenous TSGs but also the basally silenced promoters driving a GFP based 531 reporter gene in multiple colon cancer cells (Raynal et al. 2016). Most of these 532 pharmacological drugs were cardiac glycosides that did not alter DNA CpG-methylation 533 locally or histone modifications (acetylation and methylation) globally, but instead 534 affected calcium signaling by altering calcium-calmodulin kinase II (CamKII) activity 535 thereby causing nuclear exclusion of MeCP2. Further inhibition of CamKII activity 536 prevented gene reactivation and rescued drug induced cancer cell toxicity and killing 537 (Raynal et al. 2016). These findings indicate that calcium signaling pathway can be 538 targeted to prevent MeCP2-mediated TSG silencing in colon cancer.

\section{Involvement in Prostate cancer}

540 Prostate cancer remains to be the first and most common cancer affecting men in the 541 United States and is a disease of older men (Mehta and Armstrong 2016). In a majority 542 of prostate cancers, glutathione S-transferase 1 (GSTP1) gene promoter is subjected to 543 hypermethylation and inactivation. While studying epigenetic regulation of GSTP1 gene, 544 Clark group has discovered that (Stirzaker et al. 2004); firstly, histone H3 acetylation is 545 independent of gene expression. Secondly, seeds of DNA methylation promotes histone 546 deacetylation. Thirdly, spreading of DNA hypermethylation across CpG islands is not 
547 associated to MeCP2 but MBD2 binding and finally, $\mathrm{H} 3 \mathrm{~K} 9 \mathrm{me} 2$ histone methylation 548 ensues only after histone deacetylation and is linked to hypermethylation of CpG 549 islands. Such findings provide insight into the molecular and mechanistic events 550 responsible for aberrant hypermethylation of CpG-islands and hence suppression of 551 such TSGs in prostate cancer cells.

552 Inhibition of MeCP2 expression has been shown to prevent growth in prostate cancer 553 cells, while its ectopic expression confers growth advantage (Bernard et al. 2006). Such 554 over-expression of MeCP2 allows androgen dependent LNCaP cells to grow and 555 maintain the tumorigenic phenotype in absence of androgen stimulation by preserving a 556 constant level of c-Myc during an androgen depleted condition. Furthermore, MeCP2 557 over-expressing LNCaP cells were demonstrated to have a functional p53 pathway and 558 retain their sensitivity to chemotherapeutic drugs (Bernard et al. 2006).

559 Tissue inhibitor of matrix metalloproteinase-2 (TIMP2) involved in cell growth and 560 invasion, is a TSG mostly expressed in normal tissues but silenced in glioblastomas and 561 lung tumors (Mohanam et al. 1995). TIMP2 has a CpG methylated gene promoter and 562 remains suppressed in prostate cancer cells while is expressed is normal prostate 563 epithelial cells (Pulukuri et al. 2007). ChIP assay revealed presence of MeCP2 at 564 methylated TIMP-2 gene promoter in prostate cancer cells, while absent in normal cells 565 and combinatorial treatment of decitabine and TSA restored its expression and as a 566 consequence resulted in reduced tumor cell invasion. Prostate tumors expressing low 567 TIMP-2 levels showed CpG methylation of its gene promoter (Pulukuri et al. 2007). 568 These findings indicate that reduced TIMP-2 gene expression is linked to its promoter 
569 CpG methylation and MeCP2 localization, and that this may play a key role in 570 progression of prostate cancer during invasion and metastasis.

571 MeCP2-knockdown in PC3 prostate cancer cells results in low proliferating cells 572 compared to parental cells or those that are deficient in MBD1. MeCP2 deficient cells 573 are also associated with elevated apoptosis while MBD1-deficient and parental cell 574 show almost on par apoptosis (Yaqinuddin et al. 2008). Assays such as the Boyden 575 chamber invasion and wound healing migration assays reveal that MeCP2-knockdown 576 cells were both less invasive and migratory than MBD1-deficient cells. mRNA 577 expression profiles of MeCP2- and MBD1-depleted cells show significant differences 578 when compared to each other, and also when compared to the control (Yaqinuddin et 579 al. 2008). Thus, MeCP2 and MBD1 have non-redundant functions in prostate cancer 580 cells, or that their gene suppression affects independent cellular processes, and each 581 protein could regulate distinct sets of genes involved in proliferation, invasion, migration 582 and apoptosis.

583 Growth arrest and DNA damage inducible, alpha (GADD45alpha) plays a key role in 584 DNA repair, cell cycle control and apoptosis (Zhan 2005). GADD45alpha expression in 585 PC3 cells is higher than that in LNCaP and DU145 cells by 12- and 17-fold respectively 586 (Ramachandran et al. 2009). An inverse correlation exists between GADD45alpha 587 expression and methylation at 5' four $\mathrm{CpG}$ region in the proximal promoter of the gene. 588 Reactivation of GADD45alpha expression has been seen in DU145 and LNCaP cells 589 following pharmacological inhibition of DNA methylation. MeCP2 localizes to the 4 CpG 590 region in DU145 cells and its knockdown leads to increased GADD45alpha expression. 591 Increased GADD45alpha expression via either 5-azacytidine pre-treatment or by 
592 recombinant GADD45alpha expression, leads to increased sensitivity to docetaxel 593 (Ramachandran et al. 2009). Hence GADD45alpha is epigenetically regulated and 594 serves as a potential target for treatment against prostate cancer.

595 A recent study aimed at investigating the importance of green tea polyphenols (GTPs) 596 showed that human prostate cancer LNCaP cells when exposed to GTPs $(1-10 \mu \mathrm{g} / \mathrm{ml})$ 597 for 1-7 days results in GSTP1 re-expression in a concentration- and time-dependent 598 manner which was associated with decreased DNMT1 activity (Pandey, Shukla, and 599 Gupta 2010). Time-dependent GTP treatment of LNCaP cells results in decreased 600 transcript and protein levels of HDAC 1-3; MBD1, MBD4 and MeCP2, and increased 601 levels of acetylated histones H3 and H4. Exposing LNCaP cells to GTP does not lead to 602 global hypomethylation as indicated by methylation-specific PCR results for LINE-1 603 promoter, infact maintains genomic integrity as prometastatic gene S100P expression is 604 decreased in contrast to treatment with decitabine that results in global hypomethylation 605 and increased S100P expression (Pandey, Shukla, and Gupta 2010). GTP thus acts as 606 a chemotherapeutic agent due to lack of its toxicity and ability to alter DNA methylation 607 and histone modifications.

608 In DU145 cells it has been demonstrate that MeCP2 binds to the methylated target 609 gene promoters, Cyclin D2 and GLIPR1, and that this binding is lost following treatment 610 with decitabine (Muller et al. 2010). Above studies thus accentuate that epigenetic 611 deregulation in prostate cancer involves aberrant enrichment of MeCP2 at 612 hypermethylated gene promoters and that either removal of CpG methylation or 613 targeting MeCP2 itself is key to regain TSG re-expression and decreasing cancer 614 growth. 
615 Exposing LNCaP cells to curcumin (CUR) has been demonstrated to result in 616 demethylation of the proximal $14 \mathrm{CpG}$ sites of Neurog1 gene and result in the 617 restoration of its expression (Shu et al. 2011). While having limited effects on the 618 expression of MBD proteins; MBD2, MeCP2, and DNMT proteins; DNMT1 and 619 DNMT3a, CUR exposure led to significantly reduced binding of MeCP2 at Neurog1 620 gene promoter and had varying effects on the expression of HDACs, but decreased the 621 total HDAC activity. Cur treatment resulted in decreased H3K27me3 binding at the 622 Neurog1 gene promoter as well as a decrease at the global level in LNCaP cells (Shu et 623 al. 2011).

624 MeCP2 knockdown in PC3, LNCaP, and normal murine NIH3T3 cells results in a 625 defective cell cycle progression and a significant decrease in cellular proliferation, 626 together with cellular aggregation in S/G2M phase of cell cycle without leading to either 627 a severe apoptosis- and/ or senescence-like outcome (Babbio et al. 2012). MeCP2628 depleted PC3 cells show substantial decrease in lamin A, lamin B1, lamin C and lamin 629 B receptor (LBR), and immunofluorescence microscopy on such cells demonstrate the 630 appearance of an altered nuclear lamina and nuclear rim (Babbio et al. 2012). Such 631 results indicate that cells deficient in MeCP2 lose their ability to correctly assemble their 632 nuclear envelope (NE) as they have decreased levels of NE components, like lamins 633 and LBR, and hence are associated with decreased cellular proliferation and viability. 634 MeCP2 may thus potentially act as a "bridge" between chromatin and NE, facilitating 635 stability of the chromatin at nuclear periphery.

636 Exposure of LNCaP and DU145 cells with sulforaphane (SFN), an organosulfur 637 compound rich in cruciferous vegetables results in decreased activity and expression of 
638 human telomerase reverse transcriptase (hTERT), the catalytic subunit of telomerase, 639 that is associated with alterations at the hTERT promoter region in chromatin dynamics 640 and compaction involving histone PTMs, H3K4me2 and H3K18Ac (Abbas et al. 2016), 641 linked with prostate cancer recurrence (Seligson et al. 2005). ChIP-qPCR indicated that 642 MeCP2 occupancy is elevated at hTERT promoter region with increased nucleosomal 643 density following SFN treatment (Abbas et al. 2016). These results underscore the role 644 SFN mediates in suppression of hTERT in prostate cancer cells and the inherent 645 capacity of such organosulfur compounds to change the chromatin dynamics and 646 provide a novel dietary approach by which phytochemicals provide chemoprevention.

\section{Summary}

648 This review highlights various intricate roles MeCP2 plays in different cellular contexts.

649 Owing to its unusual structural features and continually increasing list of PTMs and 650 candidate binding partners, MeCP2 can differentially regulate gene expression. There is 651 a slow-yet steady increase in appreciation that such features of MeCP2 are exploited by 652 cancer cells. The dire consequence of elevating or suppressing MeCP2's role in 653 pathogenic condition like cancer mentioned herein, make them attractive targets for 654 therapeutic intervention. Nonetheless, MeCP2's involvement in the basic regulatory 655 aspects in different tissues such as the brain, becomes a limitation. There is thus an 656 increasing need for gaining a deeper comprehension of MeCP2 biology.

\section{References}

658

659

660

661

662

663
Abbas, A., J. A. Hall, W. L. Patterson, 3rd, E. Ho, A. Hsu, F. Al-Mulla, and P. T. Georgel. 2016. 'Sulforaphane modulates telomerase activity via epigenetic regulation in prostate cancer cell lines', Biochem Cell Biol, 94: 71-81.

Adams, V. H., S. J. McBryant, P. A. Wade, C. L. Woodcock, and J. C. Hansen. 2007. 'Intrinsic disorder and autonomous domain function in the multifunctional nuclear protein, MeCP2', J Biol Chem, 282: 15057-64. 
664

665

666

667

668

669

670

671

672

673

674

675

676

677

678

679

680

681

682

683

684

685

686

687

688

689

690

691

692

693

694

695

696

697

698

699

700

701

702

703

704

705

706

707

708

709

710

Agarwal, N., T. Hardt, A. Brero, D. Nowak, U. Rothbauer, A. Becker, H. Leonhardt, and M.C. Cardoso. 2007. 'MeCP2 interacts with HP1 and modulates its heterochromatin association during myogenic differentiation', Nucleic Acids Res., 35: 5402-08.

Amir, R. E., I. B. Van den Veyver, M. Wan, C. Q. Tran, U. Francke, and H. Y. Zoghbi. 1999. 'Rett syndrome is caused by mutations in X-linked MECP2, encoding methyl-CpG-binding protein 2', Nat Genet, 23: $185-8$.

Arabsolghar, R., T. Azimi, and M. Rasti. 2013. 'Mutant p53 binds to estrogen receptor negative promoter via DNMT1 and HDAC1 in MDA-MB-468 breast cancer cells', Mol Biol Rep, 40: 2617-25.

Ausio, J., A. Martinez de Paz, and M. Esteller. 2014. 'MeCP2: the long trip from a chromatin protein to neurological disorders', Trends Mol Med, 20: 487-98.

Babbio, F., I. Castiglioni, C. Cassina, M. B. Gariboldi, C. Pistore, E. Magnani, G. Badaracco, E. Monti, and I. M. Bonapace. 2012. 'Knock-down of methyl CpG-binding protein 2 (MeCP2) causes alterations in cell proliferation and nuclear lamins expression in mammalian cells', BMC Cell Biol, 13: 19.

Baker, S. A., L. Chen, A. D. Wilkins, P. Yu, O. Lichtarge, and H. Y. Zoghbi. 2013. 'An AT-hook domain in MeCP2 determines the clinical course of Rett syndrome and related disorders', Cell, 152: 984-96.

Ballestar, E., M.F. Paz, L. Valle, S. Wei, M.F. Fraga, J. Espada, J.C. Cigudosa, T.H. Huang, and M. Esteller. 2003. 'Methyl-CpG binding proteins identify novel sites of epigenetic inactivation in human cancer', EMBO J., 22: 6335-45.

Bellini, E., G. Pavesi, I. Barbiero, A. Bergo, C. Chandola, M. S. Nawaz, L. Rusconi, G. Stefanelli, M. Strollo, M. M. Valente, C. Kilstrup-Nielsen, and N. Landsberger. 2014. 'MeCP2 post-translational modifications: a mechanism to control its involvement in synaptic plasticity and homeostasis?', Front Cell Neurosci, 8: 236.

Ben-Shachar, S., M. Chahrour, C. Thaller, C. A. Shaw, and H. Y. Zoghbi. 2009. 'Mouse models of MeCP2 disorders share gene expression changes in the cerebellum and hypothalamus', Hum Mol Genet, 18: 2431-42.

Bernard, D., J. Gil, P. Dumont, S. Rizzo, D. Monte, B. Quatannens, D. Hudson, T. Visakorpi, F. Fuks, and Y. de Launoit. 2006. 'The methyl-CpG-binding protein MECP2 is required for prostate cancer cell growth', Oncogene, 25: 1358-66.

Beyer, K. S., F. Blasi, E. Bacchelli, S. M. Klauck, E. Maestrini, and A. Poustka. 2002. 'Mutation analysis of the coding sequence of the MECP2 gene in infantile autism', Hum Genet, 111: 305-9.

Billard, L. M., F. Magdinier, G. M. Lenoir, L. Frappart, and R. Dante. 2002. 'MeCP2 and MBD2 expression during normal and pathological growth of the human mammary gland', Oncogene, 21: 2704-12.

Chahrour, M., S. Y. Jung, C. Shaw, X. Zhou, S. T. Wong, J. Qin, and H. Y. Zoghbi. 2008. 'MeCP2, a key contributor to neurological disease, activates and represses transcription', Science, 320: 1224-9.

Chen, W. G., Q. Chang, Y. Lin, A. Meissner, A. E. West, E. C. Griffith, R. Jaenisch, and M. E. Greenberg. 2003. 'Derepression of BDNF transcription involves calcium-dependent phosphorylation of MeCP2', Science, 302: 885-9.

Cheng, J., M. Huang, Y. Zhu, Y. J. Xin, Y. K. Zhao, J. Huang, J. X. Yu, W. H. Zhou, and Z. Qiu. 2014. 'SUMOylation of MeCP2 is essential for transcriptional repression and hippocampal synapse development', J Neurochem, 128: 798-806.

Choudhary, C., C. Kumar, F. Gnad, M. L. Nielsen, M. Rehman, T. C. Walther, J. V. Olsen, and M. Mann. 2009. 'Lysine acetylation targets protein complexes and co-regulates major cellular functions', Science, 325: 834-40.

Cohen, S., H. W. Gabel, M. Hemberg, A. N. Hutchinson, L. A. Sadacca, D. H. Ebert, D. A. Harmin, R. S. Greenberg, V. K. Verdine, Z. Zhou, W. C. Wetsel, A. E. West, and M. E. Greenberg. 2011. 'Genome-wide activity-dependent MeCP2 phosphorylation regulates nervous system development and function', Neuron, 72: 72-85. 
711

712

713

714

715

716

717

718

719

720

721

722

723

724

725

726

727

728

729

730

731

732

733

734

735

736

737

738

739

740

741

742

743

744

745

746

747

748

749

750

751

752

753

754

755

756

Couvert, P., T. Bienvenu, C. Aquaviva, K. Poirier, C. Moraine, C. Gendrot, A. Verloes, C. Andres, A. C. Le Fevre, I. Souville, J. Steffann, V. des Portes, H. H. Ropers, H. G. Yntema, J. P. Fryns, S. Briault, J. Chelly, and B. Cherif. 2001. 'MECP2 is highly mutated in X-linked mental retardation', Hum Mol Genet, 10: 941-6.

Darwanto, A., R. Kitazawa, S. Maeda, and S. Kitazawa. 2003. 'MeCP2 and promoter methylation cooperatively regulate E-cadherin gene expression in colorectal carcinoma', Cancer Sci, 94: 4427.

Delcuve, G. P., M. Rastegar, and J. R. Davie. 2009. 'Epigenetic control', J Cell Physiol, 219: 243-50.

Ebert, D. H., H. W. Gabel, N. D. Robinson, N. R. Kastan, L. S. Hu, S. Cohen, A. J. Navarro, M. J. Lyst, R. Ekiert, A. P. Bird, and M. E. Greenberg. 2013. 'Activity-dependent phosphorylation of MeCP2 threonine 308 regulates interaction with NCoR', Nature, 499: 341-5.

Georgel, P. T., R. A. Horowitz-Scherer, N. Adkins, C. L. Woodcock, P. A. Wade, and J. C. Hansen. 2003. 'Chromatin compaction by human MeCP2. Assembly of novel secondary chromatin structures in the absence of DNA methylation', J Biol Chem, 278: 32181-8.

Gonzales, M. L., S. Adams, K. W. Dunaway, and J. M. LaSalle. 2012. 'Phosphorylation of distinct sites in MeCP2 modifies cofactor associations and the dynamics of transcriptional regulation', Mol Cell Biol, 32: 2894-903.

Guy, J., H. Cheval, J. Selfridge, and A. Bird. 2011. 'The role of MeCP2 in the brain', Annu Rev Cell Dev Biol, 27: 631-52.

Hagberg, B., J. Aicardi, K. Dias, and O. Ramos. 1983. 'A progressive syndrome of autism, dementia, ataxia, and loss of purposeful hand use in girls: Rett's syndrome: report of 35 cases', Ann Neurol, 14: 471-9.

Hagberg, B., and I. Witt-Engerstrom. 1986. 'Rett syndrome: a suggested staging system for describing impairment profile with increasing age towards adolescence', Am J Med Genet Suppl, 1: 47-59.

Hanefeld, F. 1985. 'The clinical pattern of the Rett syndrome', Brain Dev, 7: 320-5.

Ho, K. L., I. W. McNae, L. Schmiedeberg, R. J. Klose, A. P. Bird, and M. D. Walkinshaw. 2008. 'MeCP2 binding to DNA depends upon hydration at methyl-CpG', Mol Cell, 29: 525-31.

Horike, S., S. Cai, M. Miyano, J. F. Cheng, and T. Kohwi-Shigematsu. 2005. 'Loss of silent-chromatin looping and impaired imprinting of DLX5 in Rett syndrome', Nat Genet, 37: 31-40.

Jones, P. L., G. J. Veenstra, P. A. Wade, D. Vermaak, S. U. Kass, N. Landsberger, J. Strouboulis, and A. P. Wolffe. 1998. 'Methylated DNA and MeCP2 recruit histone deacetylase to repress transcription', Nat Genet, 19: 187-91.

Kankirawatana, P., H. Leonard, C. Ellaway, J. Scurlock, A. Mansour, C. M. Makris, L. S. th Dure, M. Friez, J. Lane, C. Kiraly-Borri, V. Fabian, M. Davis, J. Jackson, J. Christodoulou, W. E. Kaufmann, D. Ravine, and A. K. Percy. 2006. 'Early progressive encephalopathy in boys and MECP2 mutations', Neurology, 67: 164-6.

Kernohan, K. D., D. Vernimmen, G. B. Gloor, and N. G. Berube. 2014. 'Analysis of neonatal brain lacking ATRX or MeCP2 reveals changes in nucleosome density, CTCF binding and chromatin looping', Nucleic Acids Res, 42: 8356-68.

Kimura, H., and K. Shiota. 2003. 'Methyl-CpG-binding protein, MeCP2, is a target molecule for maintenance DNA methyltransferase, Dnmt1', J Biol Chem, 278: 4806-12.

Koch, C., and W. H. Stratling. 2004. 'DNA binding of methyl-CpG-binding protein MeCP2 in human MCF7 cells', Biochemistry, 43: 5011-21.

Kokura, K., S. C. Kaul, R. Wadhwa, T. Nomura, M. M. Khan, T. Shinagawa, T. Yasukawa, C. Colmenares, and S. Ishii. 2001. 'The Ski protein family is required for MeCP2-mediated transcriptional repression', J Biol Chem, 276: 34115-21. 
Lam, C. W., W. L. Yeung, C. H. Ko, P. M. Poon, S. F. Tong, K. Y. Chan, I. F. Lo, L. Y. Chan, J. Hui, V. Wong, C. P. Pang, Y. M. Lo, and T. F. Fok. 2000. 'Spectrum of mutations in the MECP2 gene in patients with infantile autism and Rett syndrome', J Med Genet, 37: E41.

Lewis, J. D., R. R. Meehan, W. J. Henzel, I. Maurer-Fogy, P. Jeppesen, F. Klein, and A. Bird. 1992. 'Purification, sequence, and cellular localization of a novel chromosomal protein that binds to methylated DNA', Cell, 69: 905-14.

Loree, J., I. Koturbash, K. Kutanzi, M. Baker, I. Pogribny, and O. Kovalchuk. 2006. 'Radiation-induced molecular changes in rat mammary tissue: possible implications for radiation-induced carcinogenesis', Int J Radiat Biol, 82: 805-15.

Magdinier, F., L. M. Billard, G. Wittmann, L. Frappart, M. Benchaib, G. M. Lenoir, J. F. Guerin, and R. Dante. 2000. 'Regional methylation of the 5 ' end CpG island of BRCA1 is associated with reduced gene expression in human somatic cells', FASEB J, 14: 1585-94.

Maunakea, A. K., I. Chepelev, K. Cui, and K. Zhao. 2013. 'Intragenic DNA methylation modulates alternative splicing by recruiting MeCP2 to promote exon recognition', Cell Res, 23: 1256-69.

Meehan, R., F. Antequera, J. Lewis, D. MacLeod, S. McKay, E. Kleiner, and A. P. Bird. 1990. 'A nuclear protein that binds preferentially to methylated DNA in vitro may play a role in the inaccessibility of methylated CpGs in mammalian nuclei', Philos Trans $R$ Soc Lond B Biol Sci, 326: 199-205.

Meehan, R. R., J. D. Lewis, and A. P. Bird. 1992. 'Characterization of MeCP2, a vertebrate DNA binding protein with affinity for methylated DNA', Nucleic Acids Res, 20: 5085-92.

Meehan, R. R., J. D. Lewis, S. McKay, E. L. Kleiner, and A. P. Bird. 1989. 'Identification of a mammalian protein that binds specifically to DNA containing methylated CpGs', Cell, 58: 499-507.

Mehta, A. R., and A. J. Armstrong. 2016. 'Tasquinimod in the treatment of castrate-resistant prostate cancer - current status and future prospects', Ther Adv Urol, 8: 9-18.

Mellen, M., P. Ayata, S. Dewell, S. Kriaucionis, and N. Heintz. 2012. 'MeCP2 binds to 5hmC enriched within active genes and accessible chromatin in the nervous system', Cell, 151: 1417-30.

Mirza, Sameer, Gayatri Sharma, Rajinder Parshad, Sidhartha Datta Gupta, Pranav Pandya, and Ranju Ralhan. 2013. 'Expression of DNA Methyltransferases in Breast Cancer Patients and to Analyze the Effect of Natural Compounds on DNA Methyltransferases and Associated Proteins', Journal of Breast Cancer, 16: 23-31.

Mnatzakanian, G. N., H. Lohi, I. Munteanu, S. E. Alfred, T. Yamada, P. J. MacLeod, J. R. Jones, S. W. Scherer, N. C. Schanen, M. J. Friez, J. B. Vincent, and B. A. Minassian. 2004. 'A previously unidentified MECP2 open reading frame defines a new protein isoform relevant to Rett syndrome', Nat Genet, 36: 339-41.

Mohanam, S., S. W. Wang, A. Rayford, M. Yamamoto, R. Sawaya, M. Nakajima, L. A. Liotta, G. L. Nicolson, W. G. Stetler-Stevenson, and J. S. Rao. 1995. 'Expression of tissue inhibitors of metalloproteinases: negative regulators of human glioblastoma invasion in vivo', Clin Exp Metastasis, 13: 57-62.

Muller, H. M., H. Fiegl, G. Goebel, M. M. Hubalek, A. Widschwendter, E. Muller-Holzner, C. Marth, and M. Widschwendter. 2003. 'MeCP2 and MBD2 expression in human neoplastic and nonneoplastic breast tissue and its association with oestrogen receptor status', Br J Cancer, 89: 1934-9.

Muller, I., F. Wischnewski, K. Pantel, and H. Schwarzenbach. 2010. 'Promoter- and cell-specific epigenetic regulation of CD44, Cyclin D2, GLIPR1 and PTEN by methyl-CpG binding proteins and histone modifications', BMC Cancer, 10: 297.

Murphy, C. G., and P. G. Morris. 2012. 'Recent advances in novel targeted therapies for HER2-positive breast cancer', Anticancer Drugs, 23: 765-76.

Nan, X., F. J. Campoy, and A. Bird. 1997. 'MeCP2 is a transcriptional repressor with abundant binding sites in genomic chromatin', Cell, 88: 471-81. 
805

806

807

808

809

810

811

812

813

814

815

816

817

818

819

820

821

822

823

824

825

826

827

828

829

830

831

832

833

834

835

836

837

838

839

840

841

842

843

844

845

846

847

848

849

850

851

852

Nan, X., J. Hou, A. Maclean, J. Nasir, M.J. Lafuente, X. Shu, S. Kriaucionis, and A. Bird. 2007. 'Interaction between chromatin proteins MECP2 and ATRX is disrupted by mutations that cause inherited mental retardation', Proc.NatI.Acad.Sci.U.S.A, 104: 2709-14.

Nan, X., R. R. Meehan, and A. Bird. 1993. 'Dissection of the methyl-CpG binding domain from the chromosomal protein MeCP2', Nucleic Acids Res, 21: 4886-92.

Nan, X., H. H. Ng, C. A. Johnson, C. D. Laherty, B. M. Turner, R. N. Eisenman, and A. Bird. 1998. 'Transcriptional repression by the methyl-CpG-binding protein MeCP2 involves a histone deacetylase complex', Nature, 393: 386-9.

Neupane, M., A. P. Clark, S. Landini, N. J. Birkbak, A. C. Eklund, E. Lim, A. C. Culhane, W. T. Barry, S. E. Schumacher, R. Beroukhim, Z. Szallasi, M. Vidal, D. E. Hill, and D. P. Silver. 2016. 'MECP2 Is a Frequently Amplified Oncogene with a Novel Epigenetic Mechanism That Mimics the Role of Activated RAS in Malignancy', Cancer Discov, 6: 45-58.

Nikitina, T., X. Shi, R. P. Ghosh, R. A. Horowitz-Scherer, J. C. Hansen, and C. L. Woodcock. 2007. 'Multiple modes of interaction between the methylated DNA binding protein MeCP2 and chromatin', Mol Cell Biol, 27: 864-77.

Pampalakis, G., E. Prosnikli, T. Agalioti, A. Vlahou, V. Zoumpourlis, and G. Sotiropoulou. 2009. 'A tumorprotective role for human kallikrein-related peptidase 6 in breast cancer mediated by inhibition of epithelial-to-mesenchymal transition', Cancer Res, 69: 3779-87.

Pandey, M., S. Shukla, and S. Gupta. 2010. 'Promoter demethylation and chromatin remodeling by green tea polyphenols leads to re-expression of GSTP1 in human prostate cancer cells', Int J Cancer, 126: 2520-33.

Pandey, Somnath, Glenn E. Simmons, Svitlana Malyarchuk, Tara N. Calhoun, and Kevin Pruitt. 2015. 'A novel MeCP2 acetylation site regulates interaction with ATRX and HDAC1', Genes Cancer, 6: 40821.

Pulukuri, S. M., S. Patibandla, J. Patel, N. Estes, and J. S. Rao. 2007. 'Epigenetic inactivation of the tissue inhibitor of metalloproteinase-2 (TIMP-2) gene in human prostate tumors', Oncogene, 26: 522937.

Qin, T., J. Si, N. J. Raynal, X. Wang, V. Gharibyan, S. Ahmed, X. Hu, C. Jin, Y. Lu, J. Shu, M. R. Estecio, J. Jelinek, and J. P. Issa. 2015. 'Epigenetic synergy between decitabine and platinum derivatives', Clin Epigenetics, 7: 97.

Ramachandran, K., G. Gopisetty, E. Gordian, L. Navarro, C. Hader, I. M. Reis, W. A. Schulz, and R. Singal. 2009. 'Methylation-mediated repression of GADD45alpha in prostate cancer and its role as a potential therapeutic target', Cancer Res, 69: 1527-35.

Rasti, M., R. Arabsolghar, Z. Khatooni, and Z. Mostafavi-Pour. 2012. 'p53 Binds to estrogen receptor 1 promoter in human breast cancer cells', Pathol Oncol Res, 18: 169-75.

Ray, B. K., S. Dhar, C. Henry, A. Rich, and A. Ray. 2013. 'Epigenetic regulation by Z-DNA silencer function controls cancer-associated ADAM-12 expression in breast cancer: cross-talk between MeCP2 and NF1 transcription factor family', Cancer Res, 73: 736-44.

Raynal, N. J., J. T. Lee, Y. Wang, A. Beaudry, P. Madireddi, J. Garriga, G. G. Malouf, S. Dumont, E. J. Dettman, V. Gharibyan, S. Ahmed, W. Chung, W. E. Childers, M. Abou-Gharbia, R. A. Henry, A. J. Andrews, J. Jelinek, Y. Cui, S. B. Baylin, D. L. Gill, and J. P. Issa. 2016. 'Targeting Calcium Signaling Induces Epigenetic Reactivation of Tumor Suppressor Genes in Cancer', Cancer Res, 76: 1494505.

Reeves, R., and M. S. Nissen. 1990. 'The A.T-DNA-binding domain of mammalian high mobility group I chromosomal proteins. A novel peptide motif for recognizing DNA structure', J Biol Chem, 265: 8573-82.

Reid, G., R. Metivier, C. Y. Lin, S. Denger, D. Ibberson, T. Ivacevic, H. Brand, V. Benes, E. T. Liu, and F. Gannon. 2005. 'Multiple mechanisms induce transcriptional silencing of a subset of genes, 
including oestrogen receptor alpha, in response to deacetylase inhibition by valproic acid and trichostatin A', Oncogene, 24: 4894-907.

Schanen, N. C., T. W. Kurczynski, D. Brunelle, M. M. Woodcock, L. S. th Dure, and A. K. Percy. 1998. 'Neonatal encephalopathy in two boys in families with recurrent Rett syndrome', J Child Neurol, 13: 229-31.

Schule, B., H. H. Li, C. Fisch-Kohl, C. Purmann, and U. Francke. 2007. 'DLX5 and DLX6 expression is biallelic and not modulated by MeCP2 deficiency', Am J Hum Genet, 81: 492-506.

Seligson, D. B., S. Horvath, T. Shi, H. Yu, S. Tze, M. Grunstein, and S. K. Kurdistani. 2005. 'Global histone modification patterns predict risk of prostate cancer recurrence', Nature, 435: 1262-6.

Sharma, D., J. Blum, X. Yang, N. Beaulieu, A. R. Macleod, and N. E. Davidson. 2005. 'Release of methyl CpG binding proteins and histone deacetylase 1 from the Estrogen receptor alpha (ER) promoter upon reactivation in ER-negative human breast cancer cells', Mol Endocrinol, 19: 1740-51.

Shin, J. E., S. H. Park, and Y. K. Jang. 2011. 'Epigenetic up-regulation of leukemia inhibitory factor (LIF) gene during the progression to breast cancer', Mol Cells, 31: 181-9.

Shu, L., T. O. Khor, J. H. Lee, S. S. Boyanapalli, Y. Huang, T. Y. Wu, C. L. Saw, K. L. Cheung, and A. N. Kong. 2011. 'Epigenetic CpG demethylation of the promoter and reactivation of the expression of Neurog1 by curcumin in prostate LNCaP cells', Aaps j, 13: 606-14.

Song, N., K. Li, Y. Wang, Z. Chen, and L. Shi. 2016. 'Lentivirusmediated knockdown of MeCP2 inhibits the growth of colorectal cancer cells in vitro', Mol Med Rep, 13: 860-6.

Stirzaker, C., J. Z. Song, B. Davidson, and S. J. Clark. 2004. 'Transcriptional gene silencing promotes DNA hypermethylation through a sequential change in chromatin modifications in cancer cells', Cancer Res, 64: 3871-7.

Suzuki, M., T. Yamada, F. Kihara-Negishi, T. Sakurai, and T. Oikawa. 2003. 'Direct association between PU.1 and MeCP2 that recruits mSin3A-HDAC complex for PU.1-mediated transcriptional repression', Oncogene, 22: 8688-98.

Tao, J., K. Hu, Q. Chang, H. Wu, N. E. Sherman, K. Martinowich, R. J. Klose, C. Schanen, R. Jaenisch, W. Wang, and Y. E. Sun. 2009. 'Phosphorylation of MeCP2 at Serine 80 regulates its chromatin association and neurological function', Proc Natl Acad Sci U S A, 106: 4882-7.

Tryndyak, V. P., O. Kovalchuk, and I. P. Pogribny. 2006. 'Loss of DNA methylation and histone H4 lysine 20 trimethylation in human breast cancer cells is associated with aberrant expression of DNA methyltransferase 1, Suv4-20h2 histone methyltransferase and methyl-binding proteins', Cancer Biol Ther, 5: 65-70.

Villard, L., A. Kpebe, C. Cardoso, P. J. Chelly, P. M. Tardieu, and M. Fontes. 2000. 'Two affected boys in a Rett syndrome family: clinical and molecular findings', Neurology, 55: 1188-93.

Wakefield, R.I., B.O. Smith, X. Nan, A. Free, A. Soteriou, D. Uhrin, A.P. Bird, and P.N. Barlow. 1999. 'The solution structure of the domain from MeCP2 that binds to methylated DNA', J.Mol.Biol., 291: 1055-65.

Weitzel, J. M., H. Buhrmester, and W. H. Stratling. 1997. 'Chicken MAR-binding protein ARBP is homologous to rat methyl-CpG-binding protein MeCP2', Mol Cell Biol, 17: 5656-66.

Xu, X., H. Jin, Y. Liu, L. Liu, Q. Wu, Y. Guo, L. Yu, Z. Liu, T. Zhang, X. Zhang, X. Dong, and C. Quan. 2012. 'The expression patterns and correlations of claudin-6, methy-CpG binding protein 2, DNA methyltransferase 1 , histone deacetylase 1 , acetyl-histone $\mathrm{H} 3$ and acetyl-histone $\mathrm{H} 4$ and their clinicopathological significance in breast invasive ductal carcinomas', Diagn Pathol, 7: 33.

Yamamoto, K., and M. Sonoda. 2003. 'Self-interaction of heterochromatin protein 1 is required for direct binding to histone methyltransferase, SUV39H1', Biochem Biophys Res Commun, 301: 287-92.

Yang, Longfei, Ying Cui, Jianjun Shen, Fang Lin, Xi Wang, Min Long, Junxia Wei, and Huizhong Zhang. 2015. 'Antitumor activity of SA12, a novel peptide, on SKBr-3 breast cancer cells via the mitochondrial apoptosis pathway', Drug Design, Development and Therapy, 9: 1319-30. 
901

902

903

904

905

906

907

908

909

910

911

912

913

914

915

916

917

918

919

920

921

922

923

924

925

$926 \quad$ Figure legends

927

928

929

930

931

932 102: 17551-8.

Yang, X. J., and E. Seto. 2008. 'Lysine acetylation: codified crosstalk with other posttranslational modifications', Mol Cell, 31: 449-61.

Yaqinuddin, A., F. Abbas, S. Z. Naqvi, M. U. Bashir, R. Qazi, and S. A. Qureshi. 2008. 'Silencing of MBD1 and MeCP2 in prostate-cancer-derived PC3 cells produces differential gene expression profiles and cellular phenotypes', Biosci Rep, 28: 319-26.

Yasui, D. H., S. Peddada, M. C. Bieda, R. O. Vallero, A. Hogart, R. P. Nagarajan, K. N. Thatcher, P. J. Farnham, and J. M. Lasalle. 2007. 'Integrated epigenomic analyses of neuronal MeCP2 reveal a role for long-range interaction with active genes', Proc Natl Acad Sci U S A, 104: 19416-21.

Yilmaz, T. U., A. Gunes, G. Posteki, and E. Okay. 2014. 'Rett syndrome with colon cancer presented with sigmoid volvulus: Report of a case', Int J Surg Case Rep, 5: 577-9.

Young, J. I., E. P. Hong, J. C. Castle, J. Crespo-Barreto, A. B. Bowman, M. F. Rose, D. Kang, R. Richman, J. M. Johnson, S. Berget, and H. Y. Zoghbi. 2005. 'Regulation of RNA splicing by the methylationdependent transcriptional repressor methyl-CpG binding protein 2', Proc Natl Acad Sci U SA,

Zhan, Q. 2005. 'Gadd45a, a p53- and BRCA1-regulated stress protein, in cellular response to DNA damage', Mutat Res, 569: 133-43.

Zhou, Z., E. J. Hong, S. Cohen, W. N. Zhao, H. Y. Ho, L. Schmidt, W. G. Chen, Y. Lin, E. Savner, E. C. Griffith, L. Hu, J. A. Steen, C. J. Weitz, and M. E. Greenberg. 2006. 'Brain-specific phosphorylation of $\mathrm{MeCP} 2$ regulates activity-dependent Bdnf transcription, dendritic growth, and spine maturation', Neuron, 52: 255-69.

Zocchi, L., and P. Sassone-Corsi. 2012. 'SIRT1-mediated deacetylation of MeCP2 contributes to BDNF expression', Epigenetics, 7: 695-700.

\section{Figure 1. Approximate representation of MeCP2 isoforms and their domain maps.}

A. MeCP2 e1 isoform is encoded by exons 1, 3 and 4 and has a translational start site (TSS) in exon 1 while MeCP2 isoform 2 is encoded by exons 2, 3 and 4 with its TSS located in exon 2. B. Domain map of MeCP2 isoforms is shown. $\mathrm{N}, \mathrm{N}$-terminal; MBD, Methyl-binding-domain: A-T Hook domain: TRD, Transcriptional repression domain: His-rich, Histidine-rich domain: Pro-rich, Proline-rich domain, C, C-terminal. 
933 Figure 2. MeCP2 is transcriptional suppressor. MeCP2 recognizes CpG-methyl 934 groups on DNA via its MBD domain and recruits co-repressor mSIN3A and HDAC1 to 935 cause histone deacetylation, chromatin compaction and hence target gene suppression.

936 Figure 3. Distribution of different post-translational modifications (PTMs) on 937 MeCP2 domain map. Approximate distribution of different PTMs over MeCP2 (e2 938 isoform) domain map is shown. Amino acids marked in black, red and blue color 939 respectively represent, sites for acetylation, phosphorylation and sumoylation. An 940 approximate amino acid scale is represented at the top of the domain map. Below the 941 domain map are candidate binding partners of MeCP2 showing minimal region required 942 for interaction (represented by horizontal lines).

943 Figure 4. MeCP2 is a transcription activator. MeCP2 interacts with CREB1 at target 944 gene promoters and provides accessibility to RNA Polymerase II to result in gene 945 expression. 


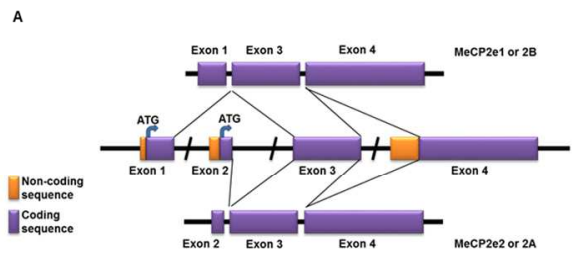

B
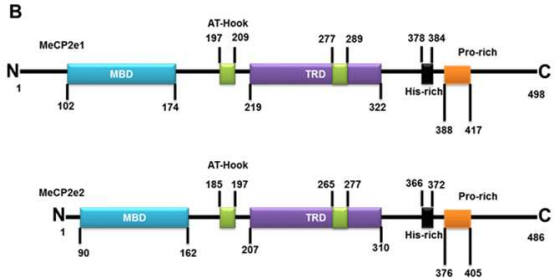

Figure 1

$338 \times 451 \mathrm{~mm}(96 \times 96$ DPI) 


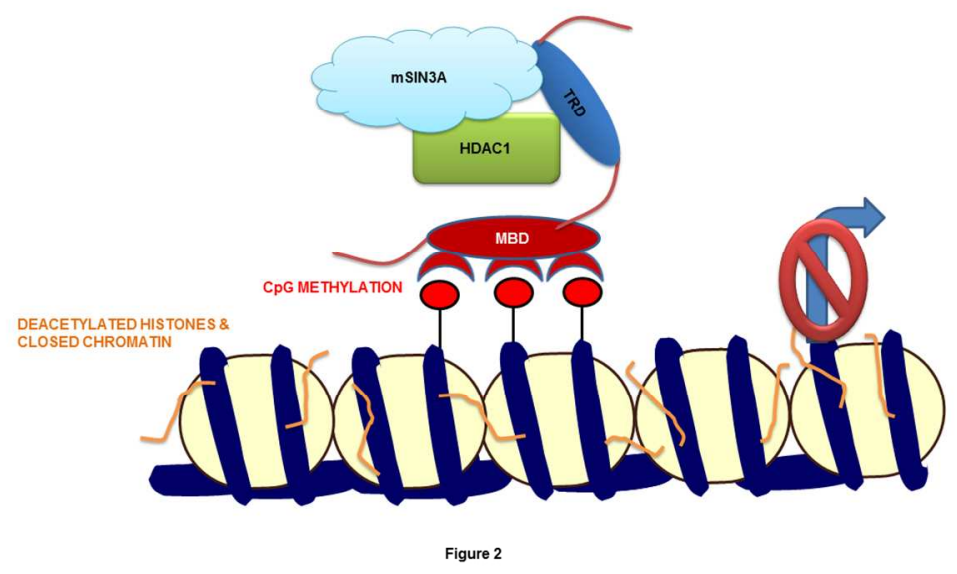

$338 \times 451 \mathrm{~mm}(96 \times 96 \mathrm{DPI})$ 


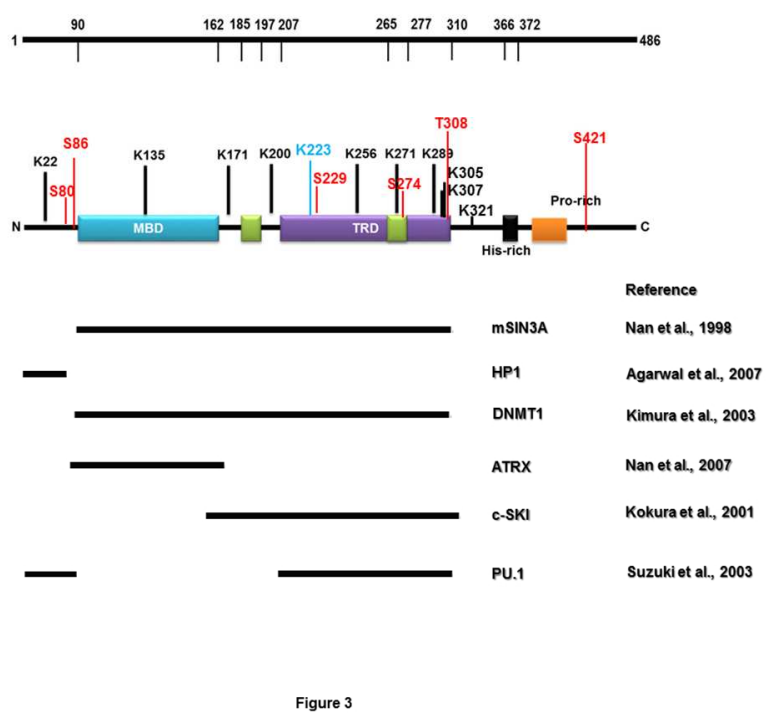

$338 \times 451 \mathrm{~mm}(96 \times 96$ DPI)

https://mc06.manuscriptcentral.com/bcb-pubs 


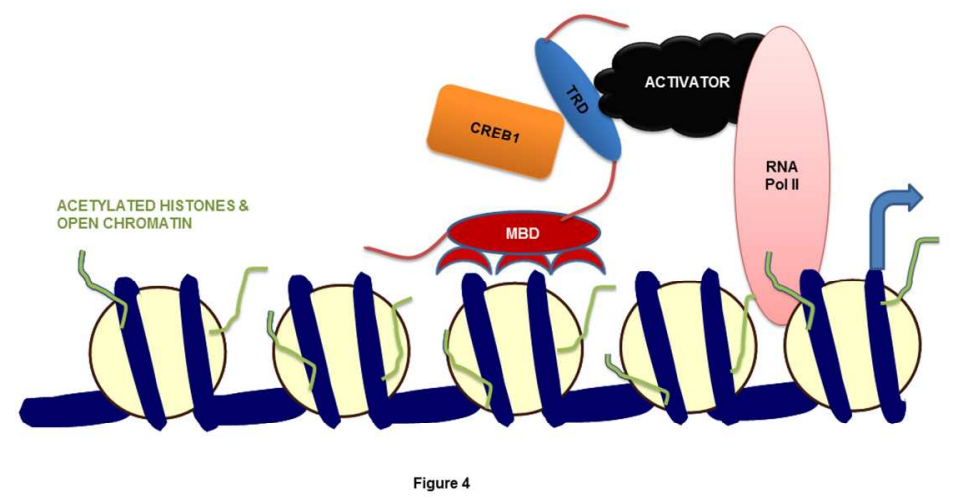

$338 \times 451 \mathrm{~mm}(96 \times 96 \mathrm{DPI})$ 